\title{
MINIMAL SOLUTIONS OF THE HEAT EQUATION AND UNIQUENESS OF THE POSITIVE CAUCHY PROBLEM ON HOMOGENEOUS SPACES
}

\author{
A. KORANYI ${ }^{1}$ AND J. C. TAYLOR ${ }^{2}$
}

\begin{abstract}
The minimal positive solutions of the heat equation on $X \times(-\infty, T)$ are determined for $X$ a homogeneous Riemannian space. A simple proof of uniqueness for the positive Cauchy problem on a homogeneous space is given using Choquet's theorem and the explicit form of these solutions.
\end{abstract}

Introduction. A minimal solution of a linear elliptic or parabolic equation is a nonnegative solution $u$ such that, whenever $0 \leqslant v \leqslant u$ is another solution, $v=\lambda u$ with some constant $0 \leqslant \lambda \leqslant 1$. By Choquet's theorem all positive solutions are convex linear combinations of minimal solutions.

It is well known that the minimal solutions of the heat equation $\Delta u=u_{t}$ of $\mathbf{R}^{n}$ are the functions $\exp \left(\|y\|^{2} t+\langle x, y\rangle\right)$ with $y \in \mathbf{R}^{n}$. In the present paper we generalise this result to a class of Riemannian manifolds with bounded geometry that includes all homogeneous Riemannian manifolds. Writing $\Delta$ for the Laplace-Beltrami operator, we show that all minimal solutions of the equation $\Delta u=u_{t}$ are of the form $u(p, t)=e^{\alpha t} f(p)$ with $f$ a minimal solution of the equation $\Delta f=\alpha f$. We note that, in the particularly interesting case of noncompact Riemannian symmetric spaces, these functions $f$ are explicitly known ([8], also [7]).

The proof of our result consists of a simple application of Moser's parabolic Harnack inequality; it is inspired by the simple proof of the theorem of Karpelevič given by Y. Guivarc'h [7]. As we shall point out, the method applies also to a large class of parabolic equations on $\mathbf{R}^{n}$.

As an application of our main result we give a simple proof of the uniqueness of the positive Cauchy problem for the heat equation on homogeneous spaces. This proof avoids the growth estimates used in the classical arguments for parabolic equations on $\mathbf{R}^{n}$.

Received by the editors May 29, 1984 and, in revised form, August 14, 1984.

1980 Mathematics Subject Classification. Primary 35K15, 43A85: Secondary 31C12, 31 C35.

$\mathrm{Key}$ words and phrases. Heat equation, minimal solutions, Cauchy problem, homogeneous space, bounded geometry.

${ }^{1}$ Partially supported by the National Science Foundation.

${ }^{2}$ Materially supported by NSERC operating grant \# A3108. 
Harnack's inequality for parabolic operators on $\mathbf{R}^{d}$. Let $L$ be a second order linear uniformly elliptic operator on $\mathbf{R}^{d}$. Assume that either

$$
L u=\sum_{i=1}^{d} \frac{\partial}{\partial x_{i}}\left(\sum_{j=1}^{n} a_{i j}(x) \frac{\partial u}{\partial x_{j}}\right)+\sum_{i=1}^{d} b_{i}(x) \frac{\partial u}{\partial x_{i}},
$$

or

$$
L u=\sum_{i, j=1}^{d} a_{i j}(x) \frac{\partial^{2} u}{\partial x_{i} \partial x_{j}}+\sum_{i=1}^{n} b_{i}(x) \frac{\partial u}{\partial x_{i}}+c(x) u,
$$

where the coefficients are measurable, for some $\lambda>0,(1 / \lambda)\|\xi\|^{2} \leqslant \sum a_{i j}(x) \xi_{i} \xi_{j} \leqslant$ $\lambda\|\xi\|^{2}$ for all $\xi \in \mathbf{R}^{d}$, and, for some $\mu>0,\left\|b_{i}\right\| \leqslant \mu$ and $-\mu \leqslant c \leqslant 0$. Let $\mathscr{L}(\lambda, \mu)$ denote the collection of operators $L$ satisfying these conditions. When $L$ is of type (1), a (weak) solution $u$ of $L u=u_{t}$ on a cylinder $\Omega \times(0, T)=D, \Omega$ open in $\mathbf{R}^{d}$, is a function such that $\|u(\cdot, t)\|_{2}$ is uniformly bounded in $t$ and has distributional derivatives $u_{x_{i}}$ with $t \leadsto\left\|u_{x_{i}}(\cdot, t)\right\|_{2} \in L^{2}(0, T)$ such that for all test functions $\varphi \in \mathscr{C}_{0}^{\infty}(D)$

$$
\iint \varphi_{t} u d x d t=\sum_{i, j=1}^{d} \iint \varphi x_{i} a_{i j} u_{x_{j}} d x d t-\sum_{i=1}^{d} \iint \varphi b_{i} u_{x_{i}} d x d t
$$

(see [14]).

For $L$ of type (2), a (weak) solution $u$ of $L u=u_{t}$ is a function in $L^{d+1}(\Omega)$, whose distributional derivatives $u_{x_{i} x_{i}}, u_{x_{i}}$ and $u_{t}$ are in $L^{d+1}(\Omega)$, such that $L u=u_{t}$ a.e. on $D$ (see [10]).

In both cases $u$ can be assumed to be continuous: in case (1) this was proved by Trudinger [14] and in case (2) it follows from the Sobolev embedding theorem.

Let $u \geqslant 0$ be a solution of $L u=u_{t}$ on $D=B(0 ; 1) \times(0, T)$ which is continuous on $\bar{D}$ (this is automatic if $L$ is of type (2)), where $B(0 ; r)=\left\{x \in \mathbf{R}^{d} \mid\|x\|<r\right\}$. Trudinger's Harnack inequality [14] in case (1) (an extension of Moser's same inequality [12]) and Krylov and Safonov's Harnack inequality [10] in case (2) state that there is a constant $C=C(T, \lambda, \mu, \theta)$ such that

$$
u(0, T) \geqslant C u(x, \theta T) \text { for all } x,\|x\| \leqslant 1 / 2 .
$$

Let $u$ be a solution of the equation $L u=u_{t}, L \in \mathscr{L}(\lambda, \mu)$, and let $w\left(\alpha x, \alpha^{2} t\right)=$ $u(x, t), \alpha \geqslant 1$. Then there is an operator $L_{\alpha} \in \mathscr{L}(\lambda, \mu)$ such that $L_{\alpha} w=w_{t}$ on the appropriate domain $\subset \mathbf{R}^{d} \times \mathbf{R}$. If $y=\alpha x$ the coefficients of $L_{\alpha}$ are $a_{i j}\left(\alpha^{-1} y\right)$, $\alpha^{-1} b_{i}\left(\alpha^{-1} y\right)$ and $\alpha^{-2} c\left(\alpha^{-1} y\right)$. Consequently, if $u \geqslant 0$ is a solution of the equation $L u=u_{t}$ for $L \in \mathscr{L}(\lambda, \mu)$, on $D_{\alpha}=B\left(0 ; \alpha^{-1}\right) \times\left(0, \alpha^{-2} T\right)$ and continuous on $\bar{D}_{\alpha}$, one has

$$
u\left(0, \alpha^{-2} T\right) \geqslant C u\left(x, \theta \alpha^{-2} T\right) \text { for all } x,\|x\| \leqslant(2 \alpha)^{-1} .
$$

These Harnack inequalities are valid even if the coefficients are also time dependent and if operators more general than those in divergence form (1) are considered (cf. Aronson [2], Trudinger [14]). 
Minimal solutions of parabolic equations on $\mathbf{R}^{d}$. Let $L$ be an operator of the type considered in $\$ 1$ and let $u \geqslant 0$ be a solution of the parabolic equation $L u=u_{t}$ on $\mathbf{R}^{d} \times \mathbf{R}$. It is said to be minimal if whenever $v$ is any other solution with $0 \leqslant v \leqslant u$ then $v=\lambda u$ for some $\lambda, 0 \leqslant \lambda \leqslant 1$. A minimal solution of $L u=\alpha u, \alpha \in \mathbf{R}$ is defined similarly.

THEOREM 1. Let $u \geqslant 0$ be a minimal solution of the parabolic equation $L u=u_{t}$ on $\mathbf{R}^{d} \times(-\infty, T)$, where $L \in \mathscr{L}(\lambda, \mu)$. Then, for some constant $\alpha, u(x, t)=e^{\alpha t} f(x)$, where $f$ is a minimal solution of the elliptic equation $L \varphi=\alpha \varphi$.

Proof. Since the coefficients of $L$ are independent of time, for any $a>0$, $v(x, t)=u(x, t-a)$ is also a solution of the equation $L u=u_{t}$ on $\mathbf{R}^{d} \times(-\infty, T)$. Let $0<a<1$. Harnack's inequality implies that there is a constant $C_{1}=C_{1}(a)$ such that $v \leqslant C_{1} u$. By minimality, there is a constant $C=C(a, u)$ such that $v=C(a, u) u$.

Hence,

$$
u(x, t)-u(x, t-a)=\{1-C(a, u)\} u(x, t) .
$$

Let $\varphi \in \mathscr{C}_{0}^{\infty}\left(\mathbf{R}^{d} \times(-\infty, T)\right)$. Then, for small $a$,

$$
\begin{aligned}
& \iint\{\varphi(x, t)-\varphi(x, t+a)\} u(x, t) d x d t \\
& \quad=\{1-C(a, u)\} \iint u(x, t) \varphi(x, t) d x d t
\end{aligned}
$$

Consequently, $-\alpha=\left.(d / d a) C(a, u)\right|_{a=0}$ exists and $-\iint \varphi_{t} u d x d t=\alpha \iint \varphi u d x d t$. As a result, $u_{t}=\alpha u$. Since the (weak) solutions are continuous, for each $x, u(x, t)=$ $e^{\alpha t} f(x)$ for all $t$.

The function $f$ is $\geqslant 0$ and a (weak) solution of $L \varphi=\alpha \varphi$ since $L u(x, t)=e^{\alpha t} L f(x)$. If $0 \leqslant g \leqslant f$ and $L g=\alpha g$ then $v(x, t)=e^{\alpha t} g(x)$ is a solution of $L v=v_{t}$. Since $0 \leqslant v \leqslant u$ it follows from the minimality of $u$ that $f$ is minimal.

REMARKS. (1) In the case of the classical heat equation $\frac{1}{2} \Delta u=u_{t}$, the minimal solutions on $\mathbf{R}^{d} \times \mathbf{R}$ are well known to be the functions $K_{y}(x, t)=\exp \left\{\|y\|^{2} t / 2+\right.$ $\langle x, y\rangle\}, y \in \mathbf{R}^{d}$. This also follows as a consequence of Theorem 1 , since $\Delta u=2 \alpha u$ has a positive solution if and only if $\alpha \geqslant 0$; and the minimal solutions when $\alpha>0$ are the exponentials $K_{b}(x, t)=\exp \sqrt{2 \alpha}\langle x, b\rangle, b \in S^{d-1}$ (cf. [9]).

(2) Agmon [1] has determined the minimal solutions of $L \varphi=\alpha \varphi$ when $L$ is in divergence form and has periodic coefficients. Hence, in this case the minimal solutions of the parabolic equation $L u=u_{t}$ are computed by Theorem 1 .

Minimal solutions of the heat equation on a homogeneous space. Let $X$ be a Riemannian manifold with a transitive isometry group; in other words, let $X$ be a homogeneous Riemannian space. Denote by $\Delta_{X}=\Delta$ the Laplace-Beltrami operator on $X$. A function $u(p, t)$, which is $\mathscr{C}^{2}$ in $p$ and $\mathscr{C}^{1}$ in $t$, is a solution of the heat equation if $\Delta u=u_{t}$. A positive solution $u$ is said to be minimal if $0 \leqslant v \leqslant u$ and $\Delta v=v_{t}$ implies $v=\lambda u$. 
THEOREM 2. Let $u \geqslant 0$ be a minimal solution of the heat equation on $X \times(-\infty, T)$, $T \in \mathbf{R}$. Then, for some constant $\alpha, u(p, t)=e^{\alpha t} f(p)$, where $f \in \mathscr{C}^{2}(X)$ is a minimal solution of the equation $\Delta f=\alpha f$. Consequently, $u(p, t)>0$ for all $(p, t) \in X \times$ $(-\infty, T)$.

Proof. Let $a>0$ and $v(p, t)=u(p, t-a)$. Then $v$ is also a solution of the heat equation on $X \times(-\infty, T)$.

Let $p_{0} \in X$. Then there is a chart $(U, \varphi)$ at $p_{0}$ and a uniformly elliptic operator $L$ on $\mathbf{R}^{d}$ of type (1) such that

$$
\left(L w-w_{t}\right)(x, t)=\left(\Delta u-u_{t}\right)(p, t)
$$

if $x=\varphi(p), p \in U, t<T$ and $w(x, t)=u(p, t)$. Hence, by Harnack's inequality (see above) there is a constant $C_{1}$ such that $u\left(p_{0}, t-a\right)=v\left(p_{0}, t\right) \leqslant C_{1} u\left(p_{0}, t\right)$ for all positive solutions $u$.

Let $g$ be an isometry of $X$. Then $w(p, t)=u\left(g^{-1} \cdot p, t\right)$ is again a solution. If $g^{-1} \cdot p=p_{0}$, it follows that $u(p, t-a)=v(p, t) \leqslant C_{1} u(p, t)$ for all $t<T$ and all positive solutions $u$. Since the isometry group is transitive, for all $p$ there is an isometry $g$ with $g \cdot p_{0}=p$. Hence, $v \leqslant C_{1} u$.

The rest of the proof is a repetition of the proof of Theorem 1 except for the last assertion.

Let $f \geqslant 0$ be such that $\Delta f=\alpha f$. Since $X$ is assumed connected, it suffices to show that the set of zeros of $f$ is open. This is a local question and is solved by Hopf's maximum principle (cf. [13, Theorem 5, p. 61, and Theorem 6, p. 64]).

REMARKS. (1) In the case of a noncompact symmetric space, the set of constants $\alpha$, for which $\Delta f=\alpha f$ has positive global solutions on $X$, is the interval $\left[-|\rho|^{2},+\infty\right)$, where $\rho$ is one-half the sum of the positive roots [8]. In this case, for any $\alpha \geqslant-|\rho|^{2}$ the minimal solutions of the equation $\Delta f=\alpha f$ have been computed by Karpelevič [8]. Hence, Theorem 2 computes the minimal solutions of the heat equation on $X \times \mathbf{R}$ oi $X \times(-\infty, T)$ for any noncompact symmetric space $X$. This settles a question raised in [11].

(2) The transitivity of the isometry group is only used to show that the local Harnack constant for a set of the type $B(x, r) \times\left(t_{1}, t_{2}\right)$, where $B(x, r)$ is an open ball about $x$, does not depend on $x$. As a result, for any positive solution $u$ of the heat equation on $X \times\left(T_{1}, T_{2}\right)$ there is a constant $C=C(a)$ such that $u(x, t-\alpha) \leqslant$ $C(a) u(x, t)$ for all $x \in X$ and $T_{1}<t-a<t<T$. A Riemannian manifold satisfying this condition will be said to have property $(\mathrm{H})$. Obviously, the proof of the theorem applies to manifolds with property $(\mathrm{H})$.

(3) As pointed out to us by N. Varopoulos, this class of manifolds includes all those manifolds with $\mathscr{C}^{1}$-bounded geometry in the sense of Cheeger et al. [3] (see p. 33). This is because in normal coordinates at $x$ the heat equation is $L u=u_{t}$ with $L$ of type (1) with $\lambda$ and $\mu$ bounded and independent of $x$.

(4) It is also obvious that the class of manifolds with property $(\mathrm{H})$ includes $N$ if $M$ satisfies $(\mathrm{H})$ and $\varphi: M \rightarrow N$ is surjective with $\Delta_{M}(u \circ \varphi)=\Delta_{N} u \circ \varphi$ for all $u \in$ $\mathscr{C}^{2}(N)$. In particular, if $N=M / \Gamma$, where $\Gamma$ is a closed subgroup of the isometry group, then $N$ satisfies (H) if $M$ does. 
Uniqueness for the positive Cauchy problem. Let $X$ be a smooth manifold and let $L-\partial / \partial t$ be a second order parabolic linear operator on $X \times(0, T)$. The positive Cauchy problem will be said to have a unique solution if the following is satisfied: when $u \geqslant 0, L u=u_{t}, u$ is continuous on $X \times[0, T)$ and $u(x, 0)=0$ for all $x \in X$, then $u \equiv 0$.

In the case $X=\mathbf{R}^{d}$ the question of uniqueness has been extensively investigated for operators of the class $\mathscr{L}(\lambda, \mu)$ where the coefficients may also depend on $t$. For $L$ in divergence form (1) (and for even more general $L$ ), Aronson [2] showed that the positive Cauchy problem has a unique solution. For $L$ in the form (2), Friedman [6] showed the same result when the coefficients were locally Hölder continuous. The arguments used involve first obtaining growth estimates for a nonnegative solution.

In the case of a rank-one noncompact symmetric space $X$, growth estimates for solutions of the heat equation are easy to make. Once the rank is greater than one, difficulties arise. As will now be shown, it is possible to completely bypass the question of growth estimates, provided one is prepared to use the Choquet theorem of integral representation for the convex cone of nonnegative solutions of the heat equation.

Theorem 3 (Uniqueness of The positive CaUchy PRoblem). Let $X$ be $a$ homogeneous Riemannian space, or, more generally, a Riemannian manifold with property $(\mathrm{H})$. Let $u \geqslant 0$ be a solution of the heat equation $\Delta u=u_{t}$ on $X \times(0, T)$. If $u$ is continuous on $X \times[0, T)$ and $u(x, 0)$ for all $x \in X$, then $u \equiv 0$.

Proof. Extend $u$ to $X \times(-\infty, T)$ by setting $u(x, t)=0$ for $t<0$. The resulting continuous function is a solution of the heat equation. To see this it suffices to note that because of the maximum principle a continuous function $u$ is a solution if and only if it agrees with the solution of the first boundary value problem on any smooth cylinder $Q=\Omega \times\left(t_{1}, t_{2}\right)$ with $\bar{Q} \subset X \times(-\infty, T)$, where the boundary value is $u$ restricted to the parabolic boundary $\partial_{p} Q=\partial \Omega \times\left[t_{1}, t_{2}\right] \cup \Omega \times\left\{t_{1}\right\}$. Alternatively, one may establish that $u$ is a solution by using a weak solution argument (cf. Aronson [2]).

The convex cone of nonnegative solutions of the heat equation on $X \times(-\infty, T)$ is a weakly complete metrisable cone in the topology of uniform convergence on compact sets (cf. [4 and 11]). Hence, every element is the barycentre of a measure carried by the extreme points of a compact convex subset (cf. [4 and 11]). It follows from Theorem 2 that $u \equiv 0$ (as a solution on $X \times(-\infty, T)$ ) since none of the minimal functions ever vanish.

Remarks. (1) The proof applies to those parabolic operators $L-\partial / \partial t$ of $\S 1$ that give rise to strict harmonic spaces in the sense of Bauer. This includes all $L$ of type (1) (even the operators considered by Aronson) and those $L$ of type (2) with time-independent Hölder continuous coefficients (cf. [4, Exercise 3.3.5]). However, the results obtained here are not as general as those of Aronson [2] and Friedman [6] since the coefficients must be time independent.

(2) The hypotheses on $u$ can probably be relaxed so as to require only that $\lim _{t \downarrow 0} u(x, t)=0$ for all $x$. This is the case for the classical heat equation on 
$\mathbf{R}^{\prime \prime} \times \mathbf{R}_{+}$. The following argument is due in part to a suggestion by $\mathbf{P}$. Koosis. By Widder's theorem $u \geqslant 0$ is represented by measure $\mu$. The measure $d \mu+d x$ represents $u+1$ and

$$
\lim _{t \downarrow 0} \frac{u}{u+1}(x, t)=0 \text { for all } x .
$$

Hence, $u /(u+1)$ tends to zero parabolically for all $x$. It follows from Doob's parabolic convergence theorem [5] that the Radon-Nikodym derivative of $d \mu$ with respect to $d \mu+d x$ is $d \mu+d x$-a.e. zero. In other words, $d \mu$ is singular with respect to $d \mu+d x$ and so $\mu=0$.

\section{REFERENCES}

1. S. Agmon, On positive solutions of elliptic equations with periodic coefficients in $\mathbf{R}^{n}$, spectral results and extensions to elliptic operators on Riemannian manifolds, Proc. Internat. Conf. on Differential Equations (Birmingham, Alabama, 1983).

2. D. G. Aronson, Nonnegative solutions of linear parabolic equations, Ann. Scuola Norm. Sup. Pisa Cl. Sci. (4) 22 (1968), 607-694.

3. J. Cheeger, M. Gromov, and M. Taylor, Finite propagation speed, kernel estimates for functions of the Laplace operator, and the geometry of complete Riemannian manifolds, J. Differential Geom. 17 (1982), 15-53.

4. C. Constantinescu and A. Cornea, Potential theory on harmonic spaces, Springer-Verlag, Berlin, 1972.

5. J. L. Doob, Relative limit theorems in analysis, J. Analyse Math. 8 (1960-61), 289-306.

6. A. Friedman, On the uniqueness of the Cauchy problem for parabolic equations, Amer. J. Math. 81 (1959), 503-511.

7. Y. Guivarc'h, Sur le représentation intégrale des fonctions harmoniques et des fonctions propres positives dans un éspace Riemannien symetrique, Bull. Sci. Math. (to appear).

8. F. I. Karpelevič, The geometry of geodesics and the eigenfunctions of the Beltrami-Laplace operator on simmetric spaces, Trans. Moscow Math. Soc. 14 (1965), 51-199.

9. A. Koranyi and J. C. Taylor, Fine convergence and parabolic convergence and parabolic convergence for the Helmholtz equation and the heat equation, Illinois J. Math. 27 (1983), 77-93.

10. N. V. Krylov and M. V. Safonov, A certain property of solutions of parabolic equations with measurable coefficients, Math. USSR-Izv. 16 (1981), 151-164 [Izv. Akad. Nauk SSSR Ser. Mat. 44 (1980)].

11. B. Mair and J. C. Taylor, Integral representation of positive solutions of the heat equation, Théorie du Potential, Lecture Notes in Math., vol. 1096, Springer-Verlag, Berlin and New York, pp. 419-433.

12. J. Moser, A Harnack inequality for parabolic differential equations, Comm. Pure Appl. Math. 17 (1964), 101-134.

13. M. H. Protter and H. F. Weinberger, Maximum principles in differential equations, Prentice-Hall, Englewood Cliffs, N. J., 1967.

14. N. Trudinger, Pointwise estimates and quasilinear parabolic equations, Comm. Pure Appl. Math. 21 (1968), 205-226.

Department of Mathematics, Washington University, St. Louis, Missouri 63130

DePariment of Mathematics, McGill University, Montreal H3A 2K6, Quebec, Canada 